\title{
Contribution des paramètres magnétiques à l'identification des niveaux stratigraphiques et de la pédogenèse (Grotte del Angel, Espagne)
}

\author{
Contribution of magnetic parameters to the identification of stratigraphic \\ levels and pedogenesis (Angel Cave, Spain)
}

\author{
A. Djerrab', I. Hedley², P. Camps³ , S. Abdessadok , C. Barroso Ruiz ${ }^{5}$, D. Botella Ortega ${ }^{6}$
}

\begin{abstract}
RÉSUMÉ
La grotte d'Angel, datée du Pléistocène moyen et de la base du Pléistocène supérieur, a livré un important matériel lithique et faunistique, et présente un intérêt exceptionnel dans la Péninsule lbérique. Vu son importance, et dans le cadre d'un programme de recherche européen (NIDPH/ER/05), une étude pluridisciplinaire a été réalisée sur le remplissage sédimentologique et les vestiges archéologiques. Nous présentons dans cet article les résultats de l'étude des propriétés magnétiques du remplissage de la grotte. Cette approche a révélé la présence de deux unités magnétostratigraphiques. L'unité stratigraphique inférieure se caractérise par une forte concentration en grains magnétiques, parmi lesquels un fort pourcentage en grains superparamagnétiques (SP). Les minéraux magnétiques sont d'origine secondaire, de petite taille, de nature douce et dominés par la magnétite et la maghémite. Ces derniers ont subi une évolution géochimique post-dépositionnelle importante (forte pédogenèse). Au contraire, dans l'unité supérieure, les valeurs des différents paramètres magnétiques sont faibles et les minéraux magnétiques sont d'origine détritique et se caractérisent par une force coercitive élevée, conforme à la présence d'hématite.
\end{abstract}

Mots clé: Grotte d'Angel, Cordoba, Espagne, susceptibilité magnétique, aimantation rémanente, oxydes de fer, pédogenèse.

\begin{abstract}
The Angel cave, dated between the middle to the base of the Upper Pleistocene, contains important lithic and faunal material, and is of exceptional interest in the lberian Peninsula. Because of its importance, and in the framework of an European research project (N'IDPH/ER/05), a multidisciplinary study including palaeomagnetism, was undertaken on the sedimentary deposit and the archaeological material. The study of the magnetic properties of the cave sediments reveals the presence of two magnetostratigraphic units. The lower stratigraphic unit is characterized by an important concentration of magnetic grains with a considerable percentage of superparamagnetic grains (SP). The magnetic minerals are of secondary origin, fine grained, magnetically soft and dominated by magnetite and maghemite. The latter have undergone an important post-depositional geochemical evolution (marked pedogenesis). On the contrary, the upper unit is characterized by weaker magnetic parameters and the magnetic minerals are harder, of detrital origin, dominated by hematite.
\end{abstract}

Keywords: Angel cave, Cordoba, Spain, magnetic susceptibility, remanent magnetization, iron oxides, pedogenesis

\footnotetext{
1 Université de Tébessa, Faculté des Sciences, Route de Constantine 12002 Tébessa, Algérie. Email: djerrab@yahoo.fr

2 Université de Genève, Département de Minéralogie, 13 rue des Maraîchers, 1205 Genève, Suisse. Musée de Préhistoire des gorges du Verdon, F-04500 Quinson. Email: ian.hedley@bluewin.ch

${ }_{3}^{3}$ Géosciences Montpellier, CNRS et Université Montpellier 2, Place Eugène Bataillon, 34095 Montpellier, France. Email: pierre.camps@gm.univ-montp2.fr

${ }^{4}$ Département de Préhistoire, Muséum National d'Histoire Naturelle, 1 rue René Panhard 75013 Paris. Email: abdess@mnhn.fr

${ }^{5}$ C/ Cantabria, 3. 29730 Rincón de La Victoria (Málaga). Email: ceciliobarroso1@hotmail.com

${ }^{6}$ Museo Arqueológico y Etnológico de Lucena (Córdoba). Email: museo@aytolucena.es
} 


\section{Introduction}

Les remplissages des grottes et des abris sous roches représentent en général d'excellentes archives géologiques et paléoclimatiques qui recèlent beaucoup d'informations sur la nature, le mode de transport et de dépôt des sédiments, ainsi que sur leur évolution physico-chimique post-dépositionnelle.

L'utilisation des paramètres magnétiques pour détecter le changement de la concentration et de la nature de grains magnétiques des sites archéologiques a été largement utilisée dans de nombreuses études (Tite \& Mullins, 1971; Peters \& Thompson, 1998; ; Marshall,1998)...etc.). En effet, les grains magnétiques sont très sensibles aux changements des conditions physico-chimiques du milieu pendant la sédimentation (Verosub \& Roberts, 1995; Evans \& Heller, 2003).

Nous proposons dans cette étude de réaliser une description exhaustive des propriétés magnétiques du remplissage de la grotte. Trois objectifs sont visés. Il s'agira: 1) de préciser la stratigraphie. 2) d'affiner les interprétations paléoclimatiques et enfin 3) de valider la présence de foyers qui parfois peuvent être confondus avec des zones riches en matière organique (Djerrab \& Camps, 2011; Djerrab \& Aifa, 2010a; Djerrab \& Hedley, 2010b).

\section{Contexte géologique, archéologique et datations}

La grotte del Angel se localise sur la commune de Lucena (fig. 1), dans la province de Córdoba en Espagne, à l'extrémité sud de la Sierra de Aras, sur le massif d'Araceli à $620 \mathrm{~m}$ d'altitude absolue. Il s'agit d'un site archéologique du Pléistocène (moyen et supérieur). La grotte est creusée dans du calcaire dolomitique d'âge Mésozoïque (Lias inférieur et moyen), qui affleure en banc massif et plonge selon la direction NNW. Ce massif a été affecté par une tectonique distensive récente, entre l'Eocène et le Miocène moyen, ayant provoqué l'apparition de fractures de direction NNE-SSW (I.G.M.E., 1991). Ces fractures ont favorisé le développement de plusieurs grottes.

Afin de déterminer la richesse archéologique, de délimiter le gisement et de proposer un plan de conservation du site, des fouilles de sauvetage ont été entamées en 1995 et se sont poursuivies durant quatre campagnes au cours desquelles d'importants vestiges archéologiques ont été mis au jour (fig. 2). Parallèlement aux fouilles, une campagne de prélèvement a été organisée durant l'été 2005 par une équipe pluridisciplinaire composée de spécialistes en magnétisme des roches, en sédimentologie et en micromorphologie.

L'étude stratigraphique menée par Abdessadok (Botella et al., 2006) montre que le remplissage de cette grotte peut être subdivisé en deux grands ensembles (fig. 3). Le premier, épais de $135 \mathrm{~cm}$, s'étend du sommet des dépôts jusqu'à l'altitude -350 $\mathrm{cm}$. Il contient peu de matériel archéologique et une fraction grossière clairsemée. Le deuxième se développe entre $-350 \mathrm{~cm}$ et la base du remplissage. Ce second ensemble comprend une grande quantité d'ossements, d'outils lithiques en silex et des fragments de calcite. Les éléments grossiers, le plus souvent de nature calcaire, sont abondants. Ce sont généralement des pierres non usées et non altérées, dont la taille varie de 1 à $10 \mathrm{~cm}$.

Au sein même de ces deux grands ensembles, plusieurs niveaux sédimentaires, définis à partir de la structure, des encroûtements ou de la colorimétrie du sédiment, sont mis en évidence (Botella et al., 2006). Ces niveaux, au nombre d'une vingtaine (fig. 3), sont surmontés par un voile stalagmitique très fin de $1 \mathrm{~cm}$ d'épaisseur (visible au sommet de la coupe transversale $\mathrm{J} / \mathrm{K})$. Ce dernier a été daté entre 100 et $120 \mathrm{ka}$ (Zouhair, 1996).

Le $1^{\text {er }}$ ensemble stratigraphique présente dans sa partie supérieure (entre -200 et $-260 \mathrm{~cm}$ ) des sédiments très friables, de couleur brun gris très foncé. La partie inférieure (entre -260 et $-350 \mathrm{~cm}$ ) débute par un niveau fortement induré de couleur brun foncé, puis se succèdent les niveaux suivants: un niveau bréchique ossifère (à $-280 \mathrm{~cm}$ ), un niveau moins induré de couleur brun rouge foncé, un niveau très friable de couleur gris rose et enfin un niveau meuble de couleur brun rouge foncé.

Le $2^{\text {ème }}$ ensemble, de couleur brun rouge très foncé, présente un encroûtement généralisé avec $70 \%$ d'éléments grossiers. La base de cet ensemble (niveau 20), sablo-limoneux, est marquée par une consistance tendre et une structure plastique des sédiments.

L'élimination du $\mathrm{CaCO}_{3}$ dans la fraction sableuse et dans la fraction limono-argileuse (Huet, 2003) améliore nettement les proportions des éléments les plus fins dans la première classe et d'une manière un peu moins perceptible dans la deuxième. Nous pensons que ces encroûtements sont syn-sédimentaires et proviendraient d'une migration horizontale des car- 


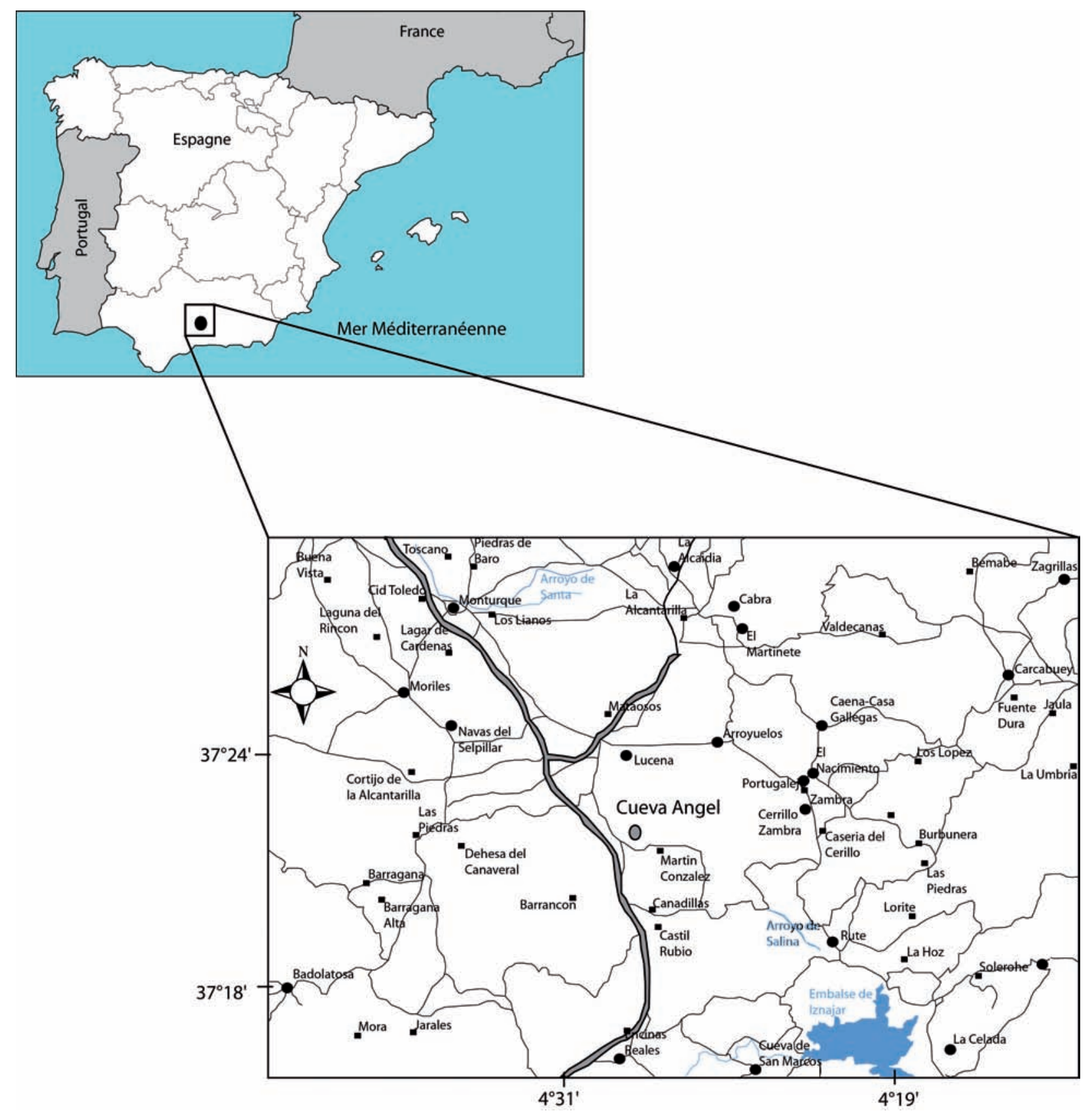

Fig. 1-Situation géographique de la grotte d'Angel.

bonates en solution puis de leur précipitation sous forme de ciment agrégeant les particules les plus fines. Celles-ci, libérées lors de l'attaque à l'acide chlorhydrique, viennent enrichir les pourcentages des sables fins et des argiles. Ces phénomènes de circulation concerneraient les deux premiers tiers de la séquence puisqu'ils montrent de nettes différences entre les fractions brute et décarbonatée. Dans le der- nier tiers, les proportions s'équilibrent, traduisant ainsi, selon notre interprétation, l'absence de la recristallisation de la calcite.

La morphoscopie des grains de quartz, effectuée par Huet (2003) sur les éléments compris entre 0,500 et $0,315 \mathrm{~mm}$, révèle que ce minéral se compose principalement de non usés et d'émoussés. Leurs pourcentages sont quasi équivalents même si l'on note, 


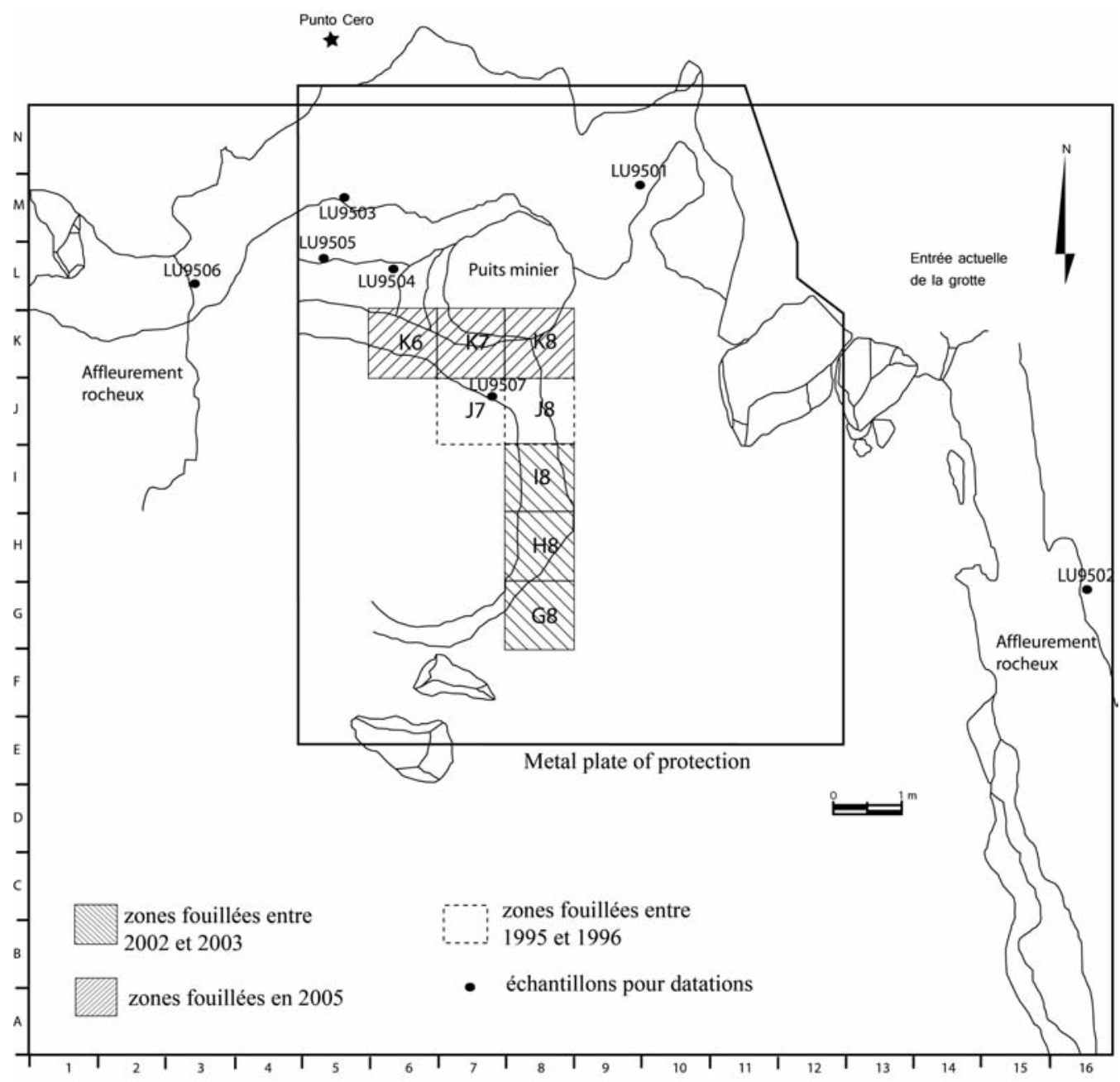

Fig. 2-Plan de la grotte d'Angel.

dans certains niveaux, de légères fluctuations au profit des non usés.

La présence en grand nombre des émoussés dans le remplissage ne serait pas liée à leur mise en place sous l'action du vent, mais à un héritage de ces grains à partir des formations géologiques et superficielles environnantes; lesquelles ont été érodées puis entraînées à l'intérieur de la grotte par des phénomènes de ruissellement. À ce titre, la constance dans les proportions des fractions granulométriques fines, tout au long de la séquence, renforce cette hypothèse et laisse supposer une provenance commune pour l'ensemble des éléments.

Zouhair (1996) a réalisé des datations radiométriques (U/Th) sur 5 échantillons géologiques (fig. 2) et 2 échantillons archéologiques, prélevés dans différents points de la grotte. Parmi les échantillons géo- logiques, deux ont été prélevés à l'extérieur de la grotte (LU9501 et LU9502) et les trois autres à l'intérieur (LU9506, LU9505 et LU9503). La série archéologique comporte uniquement deux échantillons. Le premier (LU9504) a été recueilli dans le prolongement du niveau sous-jacent à l'Unité Stratigraphique (US-VIII) et le second (LU9507) a été prélevé dans la partie sommitale de l'US I. Les datations obtenues sont présentées dans le tableau 1.

Plus de 10.000 fragments osseux, en majorité hors contexte archéologique, ont été récoltés au cours des interventions archéologiques organisées entre 1995 et 2003. La macrofaune la plus représentative de la grotte d'Angel (Botella et al., 2006) est composée de Périssodactyles (Equus sp., Equus cf. hydruntinus, Dicerorhinus cf. hemitoechus), d'Artiodactyles (Bos primigenius, Bos/Bison sp., Cervus 
Table 1. Résultats des datations absolues obtenues sur des travertins (Zouhair, 1996).

\begin{tabular}{llllll}
\hline Echantillons & $\mathrm{U} p \mathrm{ppm}$ & ${ }^{234} \mathrm{U} /{ }^{238} \mathrm{U}$ & ${ }^{230} \mathrm{Th} /{ }^{232} \mathrm{Th}$ & ${ }^{230} \mathrm{Th} /{ }^{234} \mathrm{Th}$ & Age $(\mathrm{Ka})$ \\
\hline LU9501 & 0.068 & $0.989 \pm 0.045$ & $>100$ & $1.044 \pm 0.050$ & $>400$ \\
\hline LU9502 & 0.067 & $1.008 \pm 0.045$ & 36 & $0.976 \pm 0.050$ & $394+8 /-129$ \\
\hline LU9503 & 0.071 & $1.002 \pm 0.038$ & $>100$ & $0.984 \pm 0.051$ & $446+10 /-169$ \\
\hline LU9504 & 0.146 & $1.086 \pm 0.036$ & 63 & $0.679 \pm 0.029$ & $121+11 /-10$ \\
\hline LU9505 & 0.123 & $1.030 \pm 0.026$ & 24 & $0.894 \pm 0.029$ & $237+40 /-28$ \\
\hline LU9506 & 0.052 & $1.091 \pm 0.049$ & 20 & $0.959 \pm 0.046$ & $446+200 /-63$ \\
\hline LU9507a & 0.053 & $1.050 \pm 0.044$ & $>100$ & $1.055 \pm 0.054$ & $>380$ \\
\hline LU9507b & 0.057 & $1.008 \pm 0.049$ & 22 & $1.033 \pm 0.049$ & $>369$ \\
\hline
\end{tabular}

elaphus, Sus scrofa), de Proboscidiens (Elephas sp.), de Carnivores (Ursus sp., Lynx cf. pardina) et de Lagomorphes (Oryctolagus cuniculus).

L'industrie lithique de ce site a été attribuée au Moustérien de tradition acheuléenne (Botella, 1990), attribution récemment confirmée par Botella et al. (2006).

Les actions biologique ou chimique n'ont pas eu d'effet majeur sur les dépôts, ce qui est attesté par le bon état de conservation de l'industrie et des ossements. Un grand nombre de ces derniers a été trouvé en connexion anatomique, traduisant l'absence de remaniement notable depuis leur enfouissement. Seule la recristallisation de la calcite a eu pour conséquence le développement d'encroûtements généralisés et autour des objets.

\section{Méthodologie}

L'étude des propriétés magnétiques de ce remplissage sédimentaire, peut permettre de compléter les investigations déjà réalisées par d'autres disciplines (sédimentologie, typologie, faune et datation) en apportant de nouvelles données sur la nature, le mode et les conditions de mise en place du remplissage. Les mesures et les calculs suivants ont été effectués sur 143 échantillons (placés dans des cubes en platique de $2 \mathrm{~cm}$ de côté, soit $8 \mathrm{~cm}^{3}$ ) prélevés sur la coupe stratigraphique $\mathrm{J} / \mathrm{K}$ :

(1) la susceptibilité magnétique volumique (к) est la capacité d'un corps à acquérir une aimantation lorsqu'il est soumis à un champ magnétique $H$. Cette aimantation $M$ est fonction de la minéra- logie et de la composition du sédiment en éléments magnétisables. Elle est mesurée directement sur terrain à l'aide d'un susceptibilimètre portatif (type Bartington MS2 (fréquence 958 $\mathrm{Hz}$ ) connecté à une sonde de type MS2F),

(2) la susceptibilité magnétique massique $(\chi)$, mesurée à basse fréquence $\left(\chi_{\mathrm{bf}}\right.$ à $\left.0,46 \mathrm{kHz}\right)$ et à haute fréquence $\left(\chi_{\mathrm{hf}}\right.$ à $\left.4,65 \mathrm{kHz}\right)$ (appareil MS2 connecté à une sonde MS2B, Laboratoire de Géologie de l'Université de Tébessa, Algérie),

(3) la dépendance en fréquence de la susceptibilité magnétique $\left(\chi_{\mathrm{fd}}\right)$, calculée à partir du paramètre précédent [Maher, 1986 et 1988; Mullins, 1977]. $\chi_{\mathrm{fd}}$ varie en fonction de la nature des grains magnétiques [primaires/secondaires] et en fonction de leur taille.

Exemple pour la magnétite (Dearing, 1999):

$>\chi_{\mathrm{fd}}=0$ : roches mères (métamorphiques,magmatiques, ...etc.),

$>\chi_{\mathrm{fd}}<2 \%$ : virtuellement les grains SP (superparamagnétiques) sont absents $(<10 \%)$,

$>\chi_{\mathrm{fd}}$ varie entre 2 et 10\%: présence d'une mixture de grains SP (superparamagnétiques) $(\varnothing<$ $0.005 \mu \mathrm{m}$ ) et de grains grossiers (mono-domaine « $\mathrm{MD} »$ et poly-domaine « $\mathrm{PD} »)$,

$>\chi_{\mathrm{fd}}$ varie entre 10 et 14\%: pourcentage de grains $\mathrm{SP}>$ à $75 \%$,

Les paramètres magnétiques, y compris $\chi_{\mathrm{fd}}$, sont, en général, fortement dépendant de la taille des grains. De manière à minimiser l'énergie magnétostatique, les grains sont divisés en domaines d'aimantation uniforme: (1) les grains superparamagnétiques ou SP (grains fins monodomaines dont le temps de relaxation est inférieur à la durée de l'ex- 
\begin{tabular}{l|l|l|l|l} 
E & Zone J8 & Zone J7 & Zone J6 & Zone J5
\end{tabular}$L_{-150 \mathrm{~cm}}^{\mathrm{W}}$

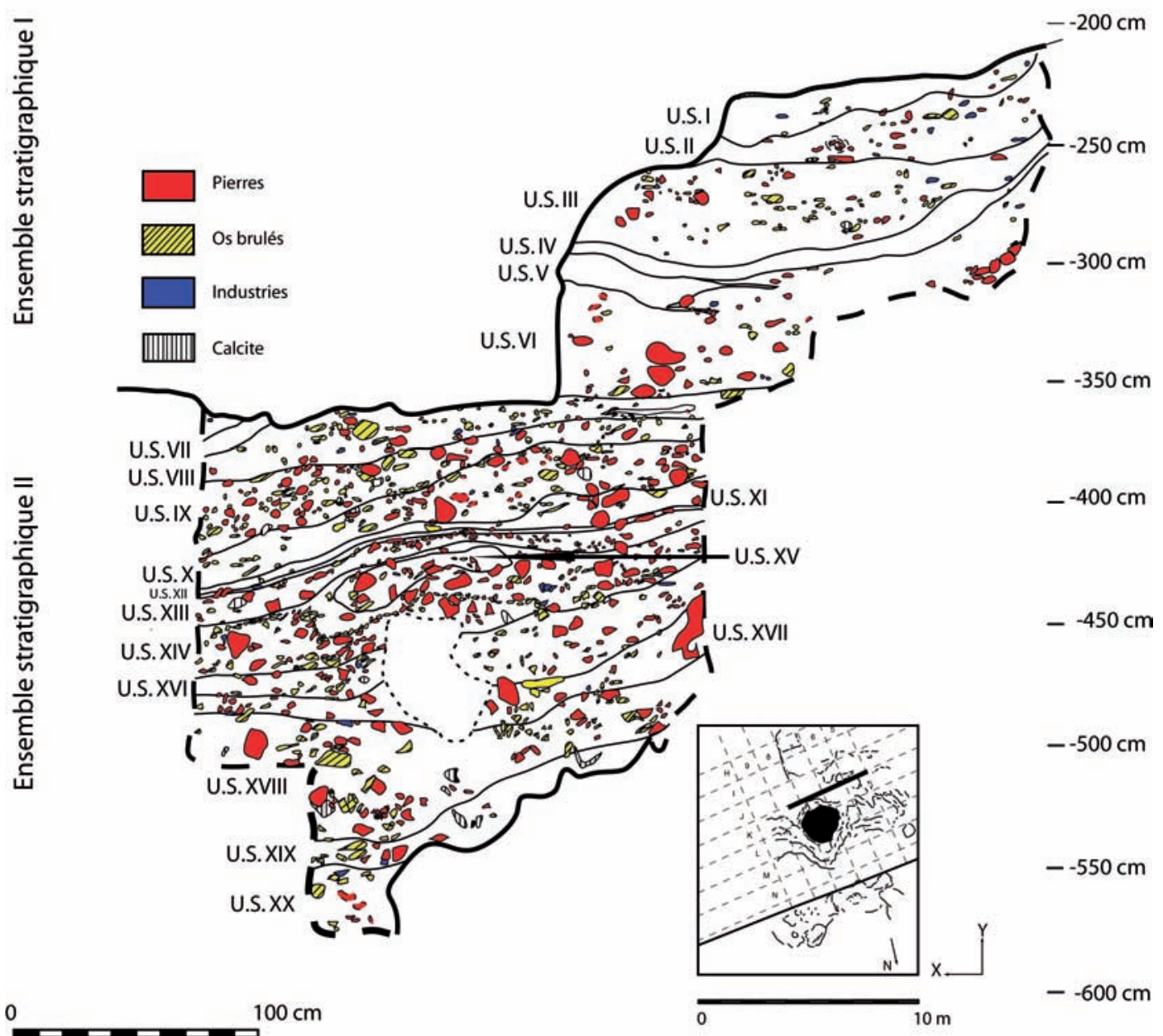

Fig. 3-Coupe stratigraphique transversale $\mathrm{J} / \mathrm{K}$ dans les bandes $5,6,7$ et dans la bande 8 à $\mathrm{x}=0$ à $50 \mathrm{~cm}$ (S. Abdessadok, 2003).

périmentation), (2) les monodomaines ou MD (un seul domaine), (3) les pseudomonodomaines ou PMD (quelques domaines; l'aimantation totale est non nulle) et (4) les polydomaines ou PD (de nombreux domaines; l'aimantation totale est nulle).

(4) l'aimantation rémanente isotherme à saturation $\left(\mathrm{ARI}_{\mathrm{s}}\right)$, induite par un électroaimant. Celui-ci permet d'appliquer un fort champ magnétique (1 T) sur l'échantillon suivant une direction déterminée, à la suite de quoi l'ARI $\mathrm{s}_{\mathrm{s}}$ est mesurée à l'aide d'un magnétomètre de type JR5 (Geofyzika), au laboratoire de magnétisme des roches de l'Université de Montpellier (France).
$\mathrm{L}^{\prime} \mathrm{ARI} \mathrm{I}_{-100 \mathrm{mT}}$ et L'ARI à palier croissant sont

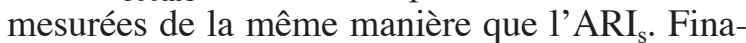
lement, une aimantation rémanente anhystéritique (ARA) de sens opposé à la direction de l'aimantation à saturation est donnée à l'échantillon en appliquant un champ constant de 0,02 mT superposé à un champ alternatif de $120 \mathrm{mT}$. L'ARA acquise est ensuite mesurée dans un magnétomètre cryogénique $2 \mathrm{G}$-Enterprises,

(5) la susceptibilité anhystérétique $\left(\kappa_{\text {ara }}\right)$ : peut être déterminée par le rapport de l'ARA sur l'intensité du champ continu inducteur (fixée à 0,02 $\mathrm{mT}$ dans notre cas), 
Table 2. Résultats de l'analyse des composantes de l'ARI.

\begin{tabular}{|c|c|c|c|c|c|c|c|c|c|c|c|c|c|}
\hline \multirow{3}{*}{ Echant. } & \multirow{3}{*}{$\begin{array}{c}\text { Unité } \\
\text { archéol. }\end{array}$} & \multicolumn{4}{|c|}{ Composante 1} & \multicolumn{4}{|c|}{ Composante 2} & \multicolumn{4}{|c|}{ Composante 3} \\
\hline & & \multicolumn{4}{|c|}{ Contribution } & \multicolumn{4}{|c|}{ Contribution } & \multicolumn{4}{|c|}{ Contribution } \\
\hline & & $(\%)$ & $\mathrm{ARI}_{\mathrm{S}}$ & $\mathrm{B} 1 / 2$ & $\mathrm{DP}$ & $(\%)$ & $\mathrm{ARI}_{\mathrm{S}}$ & $\mathrm{B} 1 / 2$ & DP & $(\%)$ & $\mathrm{ARI}_{\mathrm{S}}$ & $\mathrm{B} 1 / 2$ & $\mathrm{DP}$ \\
\hline Ang-11 & Unité-2 (-266) & 8 & 0.157 & 11.7 & 0.63 & 72 & 1.35 & 30 & 0.23 & 20 & 0.379 & 64 & 0.59 \\
\hline Ang-24 & Unité-2 (-295) & 72 & 0.735 & 26.7 & 0.23 & 28 & 0.289 & 58.1 & 0.72 & & & & \\
\hline Ang-29 & Unité-2 (-309) & 55 & 0.191 & 37.7 & 0.31 & 45 & 0.154 & 136.5 & 0.77 & & & & \\
\hline Ang-31 & Unité-2 (-313) & 3 & 0.0038 & 6.2 & 0.58 & 59 & 0.064 & 59 & 0.31 & 38 & 0.041 & 38 & 0.35 \\
\hline Ang-52 & Unité-2 (-352) & 10 & 0.037 & 28.2 & 2.21 & 57 & 0.21 & 29.2 & 0.34 & 33 & 0.128 & 58.9 & 0.37 \\
\hline Ang-85 & Unité-2 (-406) & 4 & 0.019 & 97.7 & 1.78 & 57 & 0.272 & 32.4 & 0.32 & 39 & 0.185 & 269 & 0.75 \\
\hline Ang-95 & Unité-1 (-422) & 16 & 3.64 & 14 & 0.74 & 84 & 19.8 & 21.4 & 0.28 & & & & \\
\hline Ang-105 & Unité-1 (-458) & 21 & 0.717 & 14.5 & 0.71 & 79 & 2.76 & 24.5 & 0.36 & & & & \\
\hline Ang-143 & Unité-1 (-564) & 19 & 1.57 & 14.8 & 0.64 & 81 & 6.72 & 22.4 & 0.31 & & & & \\
\hline
\end{tabular}

ARI est en $\mathrm{A} / \mathrm{m}$, DP (paramètre de dispersion) est en 10log mT et B1/2 en mT.

(6) La variation de la susceptibilité magnétique en fonction de la température est mesurée à l'aide d'un four de type CS-3 connecté au susceptibilimètre KLY-3S (AGICO, République Tchèque) au laboratoire de magnétisme des roches de l'Université de Montpellier (France).

\section{Résultats et interprétation}

\section{Unité magnétostratigraphique I}

Elle occupe la partie inférieure du remplissage (entre $-565 \mathrm{~cm}$ et $-414 \mathrm{~cm}$ en dessous du niveau zéro de la grotte). Il s'agit d'un dépôt sablo-limoneux, de couleur brun rouge très foncée, riche en matière organique et en foyers (fig. 4).

- Concentration en grains magnétiques: Trois paramètres magnétiques ont été utilisés pour estimer la concentration en grains magnétiques, à savoir $\chi_{b f}$ ou $\kappa, l^{\prime}$ 'ARI ${ }_{s}$ et l'ARA. Les valeurs de la susceptibilité magnétique volumique $(\kappa)$ sont élevées (20 à $240 * 10^{-5}$ S.I (fig. 4)), de même que celles de la susceptibilité magnétique massique mesurée à basse fréquence $\left(\chi_{\mathrm{bf}}\right.$ comprise entre 66 et $350^{*} 10^{-8} \mathrm{~m}^{3} / \mathrm{kg}$ (fig. 5.A)). Les fortes valeurs de $\kappa$ et $\chi_{b f}$ sont dues à 1 'abondance de grains magnétiques. C'est le cas également des deux paramètres magnétiques $\mathrm{ARI}_{\mathrm{s}}$ et $\mathrm{ARA}$ (fig. 5.C et
D), dont l'évolution est similaire à celle de la susceptibilité magnétique. Les fortes valeurs de ces deux paramètres témoignent de l'abondance de grains magnétiques $\left(\mathrm{ARI}_{\mathrm{s}}\right.$ comprise entre 2690 $\mathrm{mA} / \mathrm{m}$ et $11570 \mathrm{~mA} / \mathrm{m}$ et valeur moyenne de l'ARA de $250 \mathrm{~mA} / \mathrm{m}$ ).

- Taille des grains magnétiques: La dépendance en fréquence de la susceptibilité magnétique $\left(\chi_{\mathrm{fd}} \%\right.$ $=((\chi 0.47-\chi 4.7) /(\chi 4.7)) \times 100)[$ Mullins, 1973; Dearing et al., 1996] varie entre 7 et $15 \%$ (5.B) et révèle une fraction dominante de grains magnétiques de taille superparamagnétique (SP) et de grains pseudo-mono-domaine (PMD) (Eyre, 1997; Worm, 1998). La comparaison des résultats obtenus pour le rapport ARA/ARIs avec les résultats d'autres auteurs (Hartstra, 1982; Maher, 1988 et Schmidbauer \& Schembera, 1987), pour les grains de magnétite, montre que ces derniers ont une taille moyenne de $0,05 \mu \mathrm{m}$ (fig. 6).

Les valeurs faibles et constantes du rapport ARA $/ \chi_{\text {bf }}$ (aux alentours de $0.12 \mathrm{kA} / \mathrm{m}$ ) sont le résultat de la dominance de grains $\mathrm{SP}$ avec $\chi_{\mathrm{bf}}$ élevées (fig. 5.H).

- Nature des grains magnétiques: Les grains magnétiques présents saturent facilement dans un champ magnétique inférieur à $200 \mathrm{mT}$ (fig. 7, échantillons Ang-11 et Ang-143) [les valeurs du rapport $\mathrm{ARI}_{-100} / \mathrm{ARI}_{\mathrm{s}}$ sont fortes et se rapprochent de 1]. La saturation très rapide des grains 


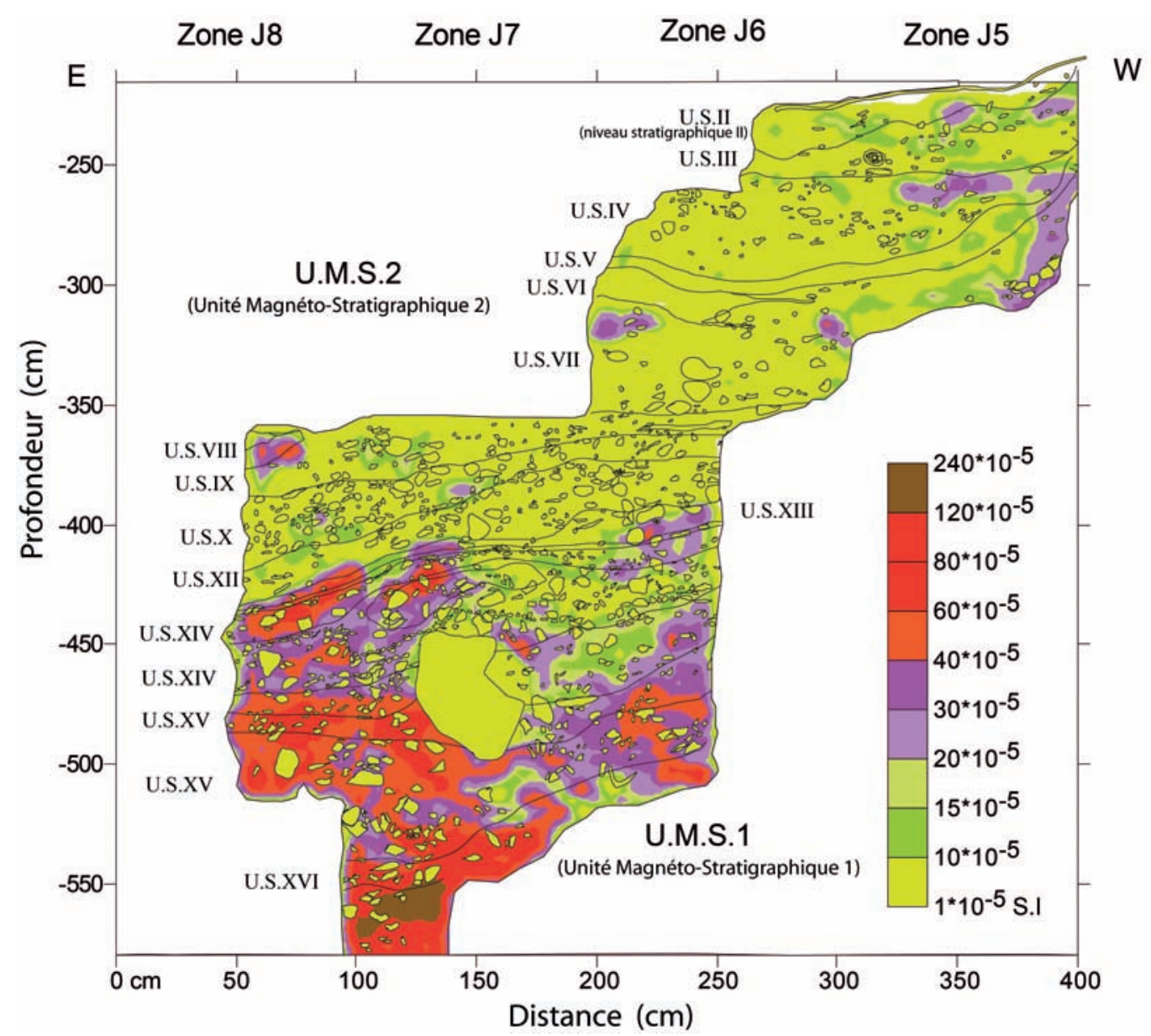

Fig. 4-Variation de la susceptibilité magnétique volumique ( $\mathrm{K}^{*} 10-5 \mathrm{~S}$.I) sur la coupe stratigraphique transversale J/K.

magnétiques entre 100 et $200 \mathrm{mT}$ est caractéristique de la dominance de grains ferrimagnétiques de faible champ coercitif tels que la magnétite (Lanci et al., 1999). Les valeurs du rapport $\mathrm{ARI}_{\mathrm{s}} / \chi_{\mathrm{bf}}$ (fig. 5.F) sont très faibles à cause de la présence de grains SP. Ces derniers ont de fortes valeurs de $\chi_{b f}$ (fig. 8). Au contraire, concernant les aimantations rémanentes, les valeurs du rapport ARA/ARI s sont élevées (forte concentration de grains de petite taille de magnétite ou de maghémite).

Chaque composante magnétique est décrite par l'étude de la courbe d'acquisition de l'aimantation à saturation $\left(\mathrm{ARI}_{\mathrm{s}}\right)$, à l'aide du paramètre $\mathrm{B}_{1 / 2}$ qui est le champ magnétique pour lequel la moitié de l'ARI $\mathrm{S}_{\mathrm{S}}$ est atteinte, et enfin à l'aide du paramètre DP, représentant la dispersion de la distribution (Robertson \& France, 1994; Kruiver et al., 2001 et 2003).
La décomposition de l'ARI de l'échantillon Ang-95 (fig. 9), prélevé à $-422 \mathrm{~cm}$, montre la présence de deux populations de grains magnétiques; la première contribue à $16 \%$ de l'ARI totale de l'échantillon et a un champ coercitif moyen de 14 mT. Cette composante est portée par les grains de taille polydomaine de magnétite ou de maghémite. La deuxième, qui contribue à $84 \%$ de l'ARI de l'échantillon, se caractérise par un champ coercitif moyen légèrement plus élevé que celui de la première composante $(21 \mathrm{mT})$, ce qui caractérise probablement des grains de maghémite ou de magnétite. Les grains magnétiques de cet échantillon saturent rapidement dans un champ magnétique faible. Le S-ratio (= (1-IRM-0.3T/IRM1T)/2) vaut 0.995 .

Les autres échantillons Ang-105 (Tab. 2) et Ang143 (fig. 9), prélevés respectivement à $-458 \mathrm{~cm}$ et à $-564 \mathrm{~cm}$, se comportent de manière identique, ce 


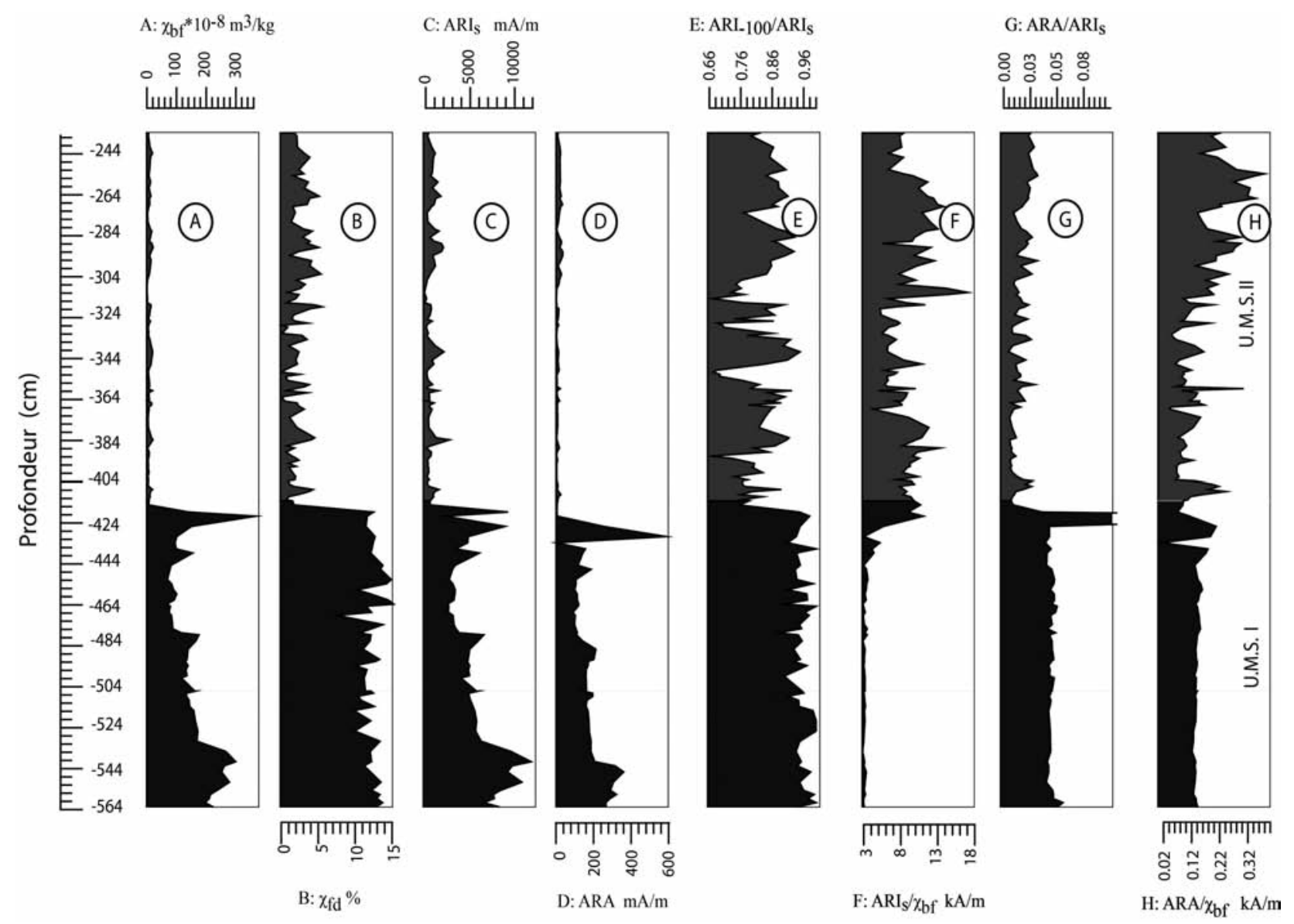

Fig. 5-Paramètres magnétiques des échantillons prélevés dans la grotte d’Angel.

qui confirme la présence de magnétite et de maghémite de taille PD et MD.

La courbe de la variation de la susceptibilité magnétique en fonction de la température de l'échantillon Ang-95 [prélevé à $-422 \mathrm{~cm}$ ] (fig. 10) montre une augmentation jusqu'à $100^{\circ} \mathrm{C}$, vraisemblablement liée à la présence de la goethite (température de Néel) (Ozdemir \& Dunlop, 2000). Les valeurs de la susceptibilité magnétique continuent à augmenter progressivement, mais plus doucement, jusqu'à $260^{\circ} \mathrm{C}$. Entre 260 et $350{ }^{\circ} \mathrm{C}$, elles augmentent fortement et témoignent de la transformation des sulfures de fer ou de lépidocrocite en maghémite. La chute de $\chi_{\text {bf }}$ vers 400 ${ }^{\circ} \mathrm{C}$ est le résultat de la décomposition de la maghémite en hématite. Enfin, les valeurs de $\chi_{\mathrm{bf}} \mathrm{s}$ 'annulent à $580{ }^{\circ} \mathrm{C}$ (point de Curie de la magnétite). Pendant le refroidissement, les valeurs de $\chi_{b f}$ diminuent fortement, indiquant la transformation d'une partie des minéraux ferromagnétiques en hématite. A basse température, nous n'observons pas la transition de Ver- wey de la magnétite comme on aurait pu s'y attendre d'après nos résultats précédents. Celle-ci a pu s'oxydée superficiellement en maghémite (Ozdemir \& Dunlop, 2010).

Sur la courbe de la variation de la susceptibilité magnétique en fonction de la température (échantillon Ang-143, prélevé à $-564 \mathrm{~cm}$ (fig. 10)), nous observons une légère diminution à $100^{\circ} \mathrm{C}$, que nous interprétons par la présence de goethite (température de Néel). A partir de $230{ }^{\circ} \mathrm{C}$, les valeurs de la susceptibilité magnétique diminuent progressivement en montrant deux points d'inflexion à $350{ }^{\circ} \mathrm{C}$ et à $520^{\circ} \mathrm{C}$, puis s'annulent à $580^{\circ} \mathrm{C}$ (point de Curie de la magnétite). Dans cet échantillon, la maghémite est le minéral le plus représenté, suivi par la magnétite.

Différents auteurs (Guo et al., 2001, Deng et al., 2000, Verosub et al., 1993) suggèrent que la maghémite contribue fortement aux valeurs de la susceptibilité magnétique et concluent qu'elle a une origine pédogénique et qu'elle s'est formée sous des condi- 


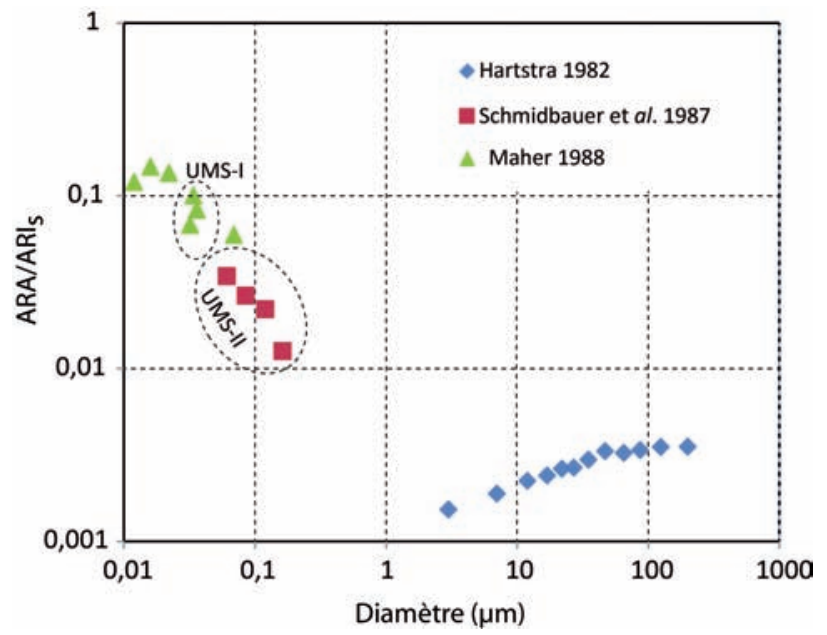

Fig. 6-Le rapport de ARA/ARls en fonction du diamètre des grains de magnétite $(\mu \mathrm{m})$.
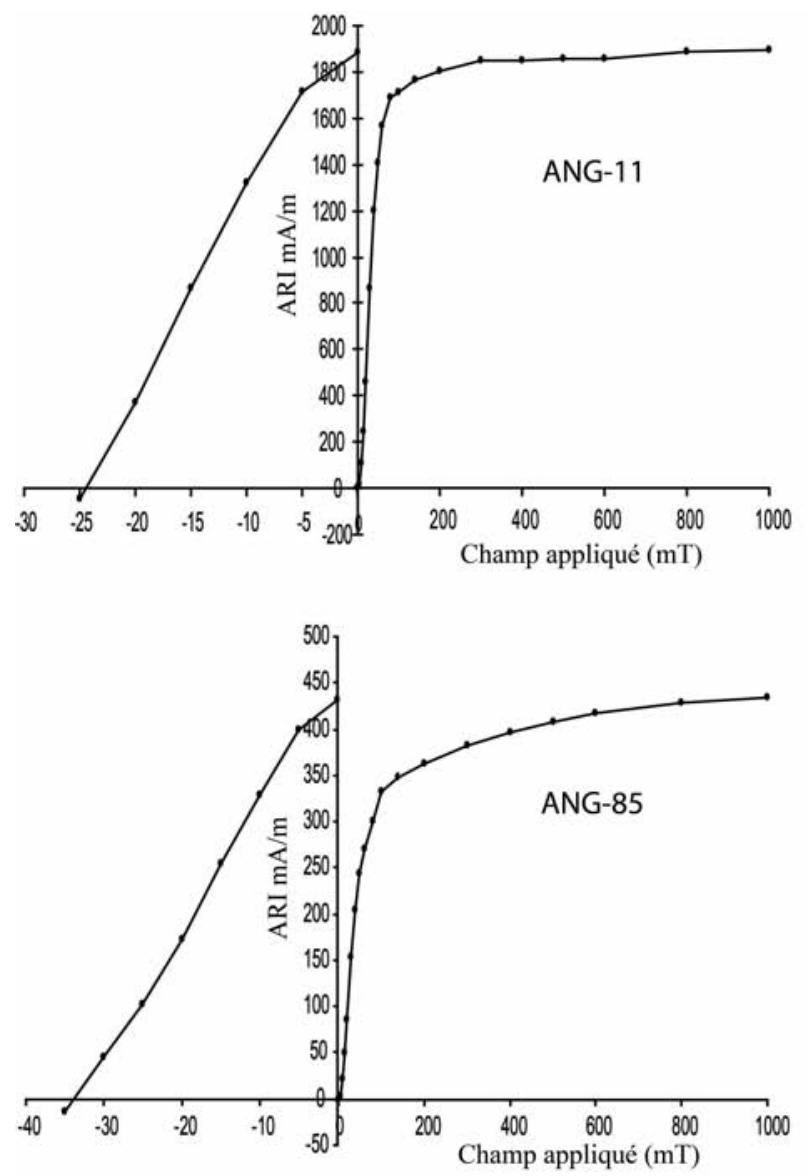

tions climatiques humides. Les plus fortes valeurs de la susceptibilité magnétique et des autres paramètres magnétiques sont enregistrées à la base du remplissage, dans des niveaux riches en foyers. L'augmentation de la température au niveau du sol entraîne la réduction de l'hématite en magnétite et la réoxydation éventuelle de celle-ci en maghémite (Le Borgne, 1960; Weston, 2004). Cette unité stratigraphique se caractérise donc par un fort pourcentage d'éléments fins SP, par une forte concentration en grains magnétiques (comme le montre la figure 8) et par un concrétionnement calcitique généralisé (les concrétions se forment sous un climat humide). Les fortes valeurs de l'ARA, du rapport ARA/ARI (Geiss \& Zanner, 2006) et les faibles valeurs du rapport $\chi_{\mathrm{bf}} / \mathrm{ARI}_{\mathrm{s}}$ témoignent en faveur d'un milieu humide et d'une forte quantité de précipitation atmosphérique.
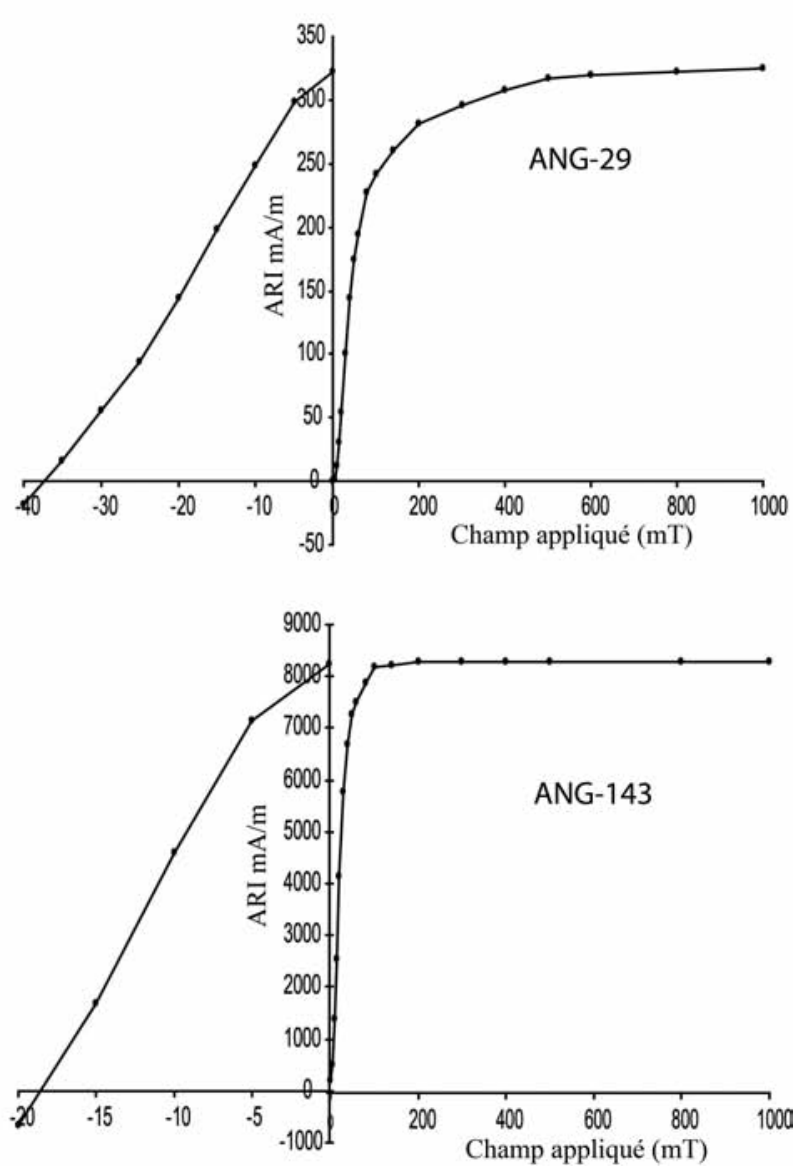

Fig. 7—Courbes d'acquisition de l'aimantation rémanente isotherme à saturation (ARIs) en fonction du champ appliqué en mT. 


\section{Unité magnétostratigraphique /I}

Elle occupe la partie supérieure du remplissage entre -414 et $-56 \mathrm{~cm}$ (fig. 3) et est caractérisée par la dominance de la fraction limono-sablonneuse. Les sédiments ont une couleur plus claire que dans la partie inférieure.

- Concentration en grains magnétiques: Dans cette unité, les valeurs de la susceptibilité magnétique chutent brutalement ( 1 à $19 * 10^{\mathrm{e}}-8$ $\mathrm{m}^{3} / \mathrm{kg}$ ) [fig. 5], ainsi que celles des deux paramètres magnétiques $\mathrm{ARI}_{\mathrm{S}}$ (fig. $5 \mathrm{C}$, valeur moyenne de $84 \mathrm{~mA} / \mathrm{m}$ ) et ARA (fig. 5D, valeur moyenne de $15 \mathrm{~mA} / \mathrm{m}$ ). La concentration en grains magnétiques est donc faible.

- Taille des grains magnétiques: La dépendance en fréquence de la susceptibilité magnétique est très faible $(0.2$ à $5 \%)$ [fig. 5.B], indiquant l'abondance de grains magnétiques d'origine détritique (primaires), ce qui est confirmé par les fai-

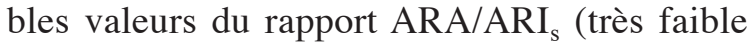
pourcentage de grains de taille mono-domaine) [fig. 5.G]. Le diagramme de ARA/ARI ${ }_{s}$ en fonction de la taille des grains de magnétite, montre que la taille moyenne de ces grains est de 0.1 $\mu \mathrm{m}$ (fig. 6).

- Nature des grains magnétiques: Dans cette unité, des valeurs du rapport ARI-100/ARI siminuent (fig. 5.E), cette diminution étant due à la dominance des oxydes de fer à champ coercitif élevé (hématite et/ou goethite) [Peters \& Thompson, 1998b]; cette affirmation renforcée par les fortes valeurs du rapport $A R I_{s} / \chi_{b f}$ (fig. 5.F).

Afin de caractériser la minéralogie magnétique par une reconstitution des populations de grains, mises en évidence à partir des spectres de coercivité, nous avons traité les données selon la méthode de Kruiver et al., (2001), reprise par Heslop et al. (2002) et Egli (2003). Les courbes de l'ARI à palier croissant des échantillons Ang-29 et Ang-85 (fig. 7) montrent que les grains magnétiques ne saturent pas à $100 \%$ en présence d'un champ de l'ordre de $1 \mathrm{~T}$, ce qui peut s'expliquer par la présence de grains magnétiques de fort champ coercitif (goethite ou hématite).

La décomposition de l'ARI de l'échantillon Ang-11, prélevé à $-266 \mathrm{~cm}$; (fig. 9) montre la présence de trois composantes magnétiques. La première contribue à $8 \%$ de la valeur totale de l'ARI de l'échantillon et présente un faible champ coercitif $(11,7 \mathrm{mT})$. Il s'agit probablement de grains de

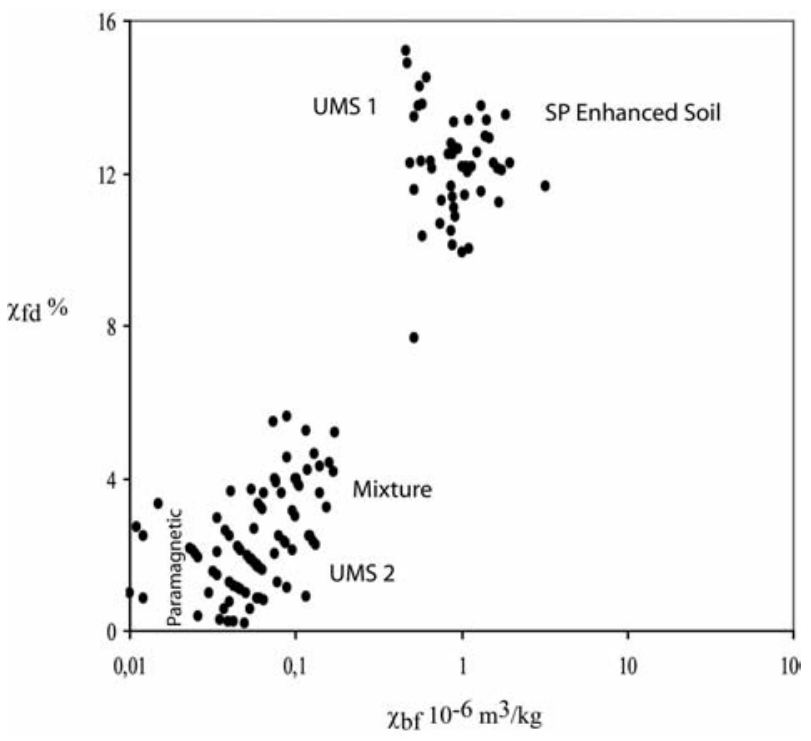

Fig. 8 - $\chi_{\mathrm{fd}} \%$ function de $\chi_{\mathrm{bf}} 10^{-6} \mathrm{~m}^{3} / \mathrm{kg}$

magnétite de taille polydomaine. La deuxième composante contribue à $72 \%$ de l'ARI totale et présente un champ coercitif de $30 \mathrm{mT}$ (magnétite de taille mono-domaine). Enfin, la troisième composante magnétique contribue à $20 \%$ de l'ARI totale et présente un champ coercitif de $64.6 \mathrm{mT}$. Cette dernière est vraisemblablement portée par les grains d'hématite.

Les échantillons Ang-24, 29, 31, 52 et 85 montrent deux à trois composantes magnétiques, parmi lesquelles l'une de fort champ coercitif (269 mT), qui pourrait correspondre à des grains d'hématite.

La courbe de la susceptibilité magnétique en fonction de la température de l'échantillon Ang-41 (fig. 10 ), prélevé à $-271 \mathrm{~cm}$, confirme la faible proportion de grains magnétiques (les valeurs de $\chi$ bf sont 50 fois inférieures à celles de l'unité I). Au-delà de $450^{\circ} \mathrm{C}$, les valeurs de $\chi_{b f}$ augmentent légèrement, ce qui pourrait caractériser le pic de Hopkinson (formation de magnétite de petite taille "monodomaine », selon Dunlop \& Ozdemir, 1997). La susceptibilité magnétique diminue fortement à $580{ }^{\circ} \mathrm{C}$ (température de Curie de la magnétite). Au-delà de $580{ }^{\circ} \mathrm{C}$, ses valeurs ne s'annulent pas et signalent vraisemblablement la présence d'hématite.

Pendant le refroidissement, des valeurs de $\chi_{b f}$ augmentent nettement entre 550 et $400{ }^{\circ} \mathrm{C}$ (elles sont 10 fois supérieures à celles de $\chi_{b f}$ pendant la chauffe), indiquant la production d'une phase magnétique pendant la chauffe par réduction. 

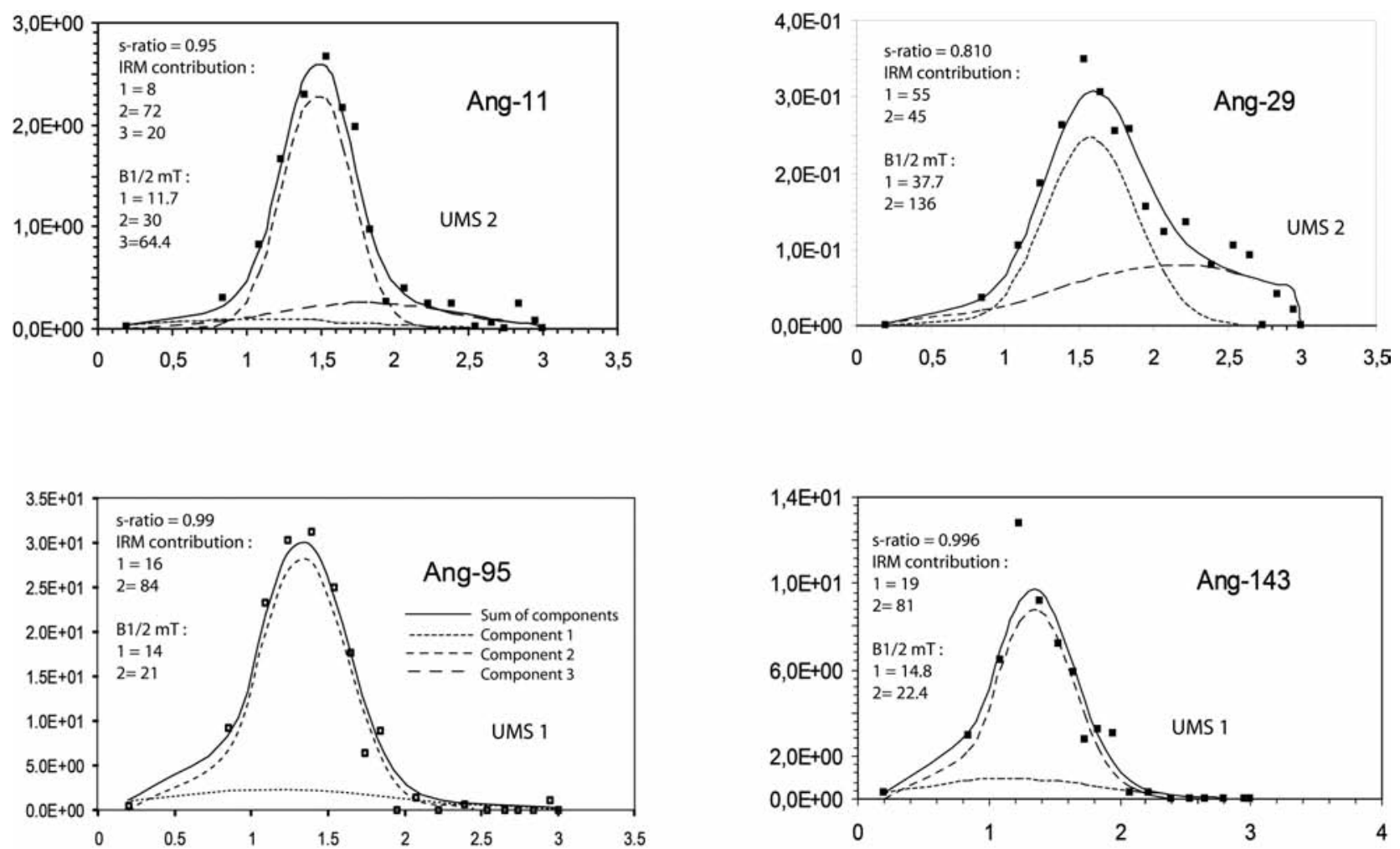

Fig. 9-Log de la distribution gaussienne cumulative (DGC) ajusté au gradient de l'acquisition de l'ARI.

A l'inverse de l'Unité I (dominée par la maghémite et la magnétite), cette unité contient un pourcentage non négligeable de grains magnétiques de fort champ coercitif (hématite et goethite), les grains de taille SP sont minoritaires et les concrétions calcitiques rares. Les très faibles valeurs de l'ARA et du rapport ARA/ARI ${ }_{s}$, ainsi que la sensibilité de l'ARA aux précipitations atmosphériques (elle augmente avec ces dernières) (Geiss \& Zanner, 2006) témoignent d'un faible taux de précipitation atmosphérique. Le climat probable était de type semi-aride proche du climat actuel, caractérisé par une alternance de périodes humides et de périodes sèches (conditions peu favorables au développement d'une forte pédogenèse).

\section{Conclusion}

Cette étude a permis de mettre en évidence deux unités magnéto-stratigraphiques bien distinctes. La partie inférieure du remplissage est caractérisée par de fortes valeurs de la susceptibilité magnétique, de la dépendance en fréquence de la susceptibilité magnétique ainsi que de l'ARI $\mathrm{s}_{\mathrm{s}}$ et l'ARA. Les grains magnétiques de cette unité stratigraphique saturent rapidement dans un champ faible, ce qui est confirmé par les valeurs du rapport $\mathrm{ARI}_{-100} / \mathrm{ARI}_{\mathrm{s}-1000}$. Ces grains magnétiques dominants sont de taille très fine (SP), suivis par les MD et les PMD de magnétite et de maghémite. Les courbes de la susceptibilité magnétique en fonction de la température confirment la dominance de magnétite et de maghémite. Les grains SP sont d'origine pédologique et se sont formés dans un milieu oxydant sous un climat humide.

Au contraire, les sédiments de l'unité supérieure présentent de faibles valeurs des différents paramètres magnétiques, ce qui indique une faible concentration en minéraux magnétiques. Ces derniers ne saturent pas rapidement même en présence d'un champ magnétique fort: il s'agit donc de grains magnétiques de fort champ coercitif (probablement de l'hématite), ce qui a été confirmé par les courbes de la décomposition de l'ARI. Les grains magnétiques d'origine pédologique sont quasiment absents dans cette unité stratigraphique (très faibles valeurs de la dépendance en fréquence de la susceptibilité 

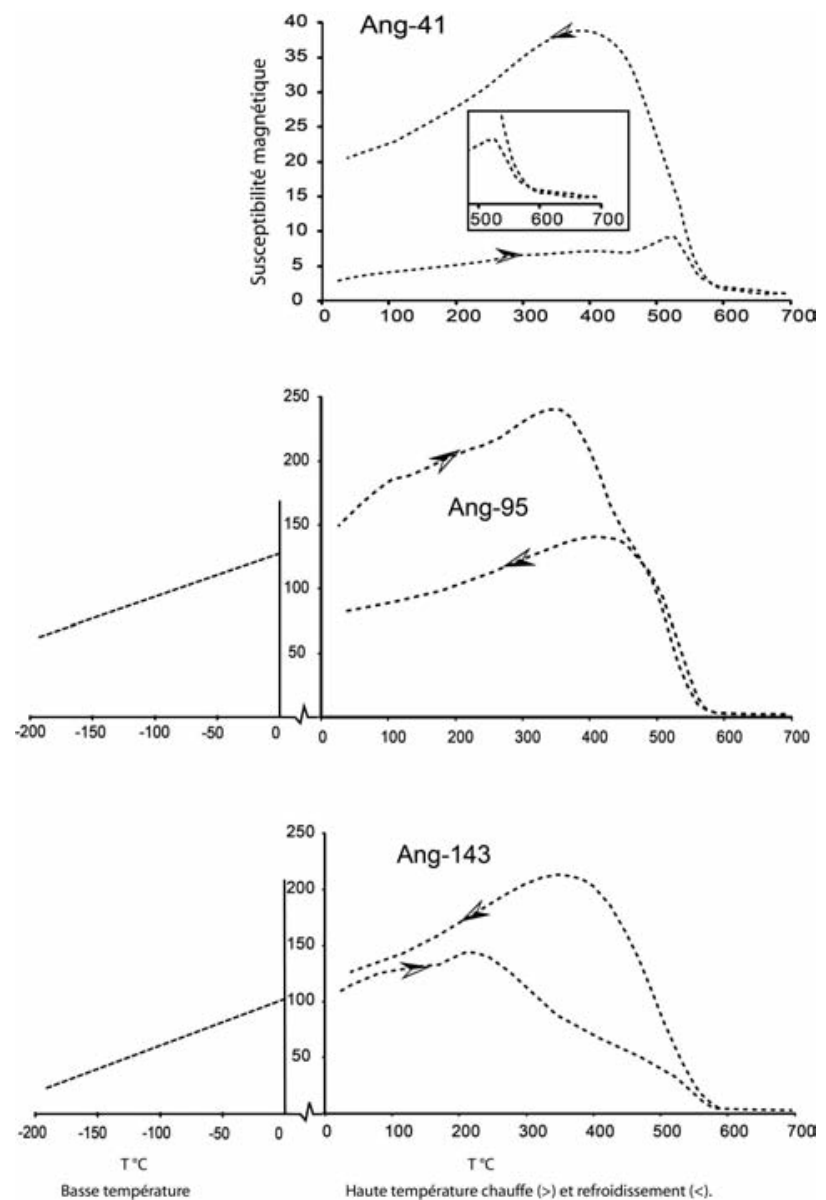

Fig. 10-Variation de la susceptibilité magnétique en fonction de la température.

magnétique), au contraire des grains d'origine détritique qui dominent. Le dépôt de cette unité s'est mis en place sous des conditions climatiques similaires à l'actuel (climat semi-aride).

\section{Références}

Botella Ortega, D. (1990). El yacimiento musteriense de la C.S.A. 1 (Lucena). Cuaderno de campo. $V^{a}$ Reunión de Campo de AEQUA-Andalucía. Ayuntamiento de Lucena. Lucena (Córdoba): 65-79.

Botella Ortega, D.; Barroso Ruiz, C.; Riquelme Cantal, J.A.; Abdessadok, S.; Caparrós, M.; Bermejo, L.V.; Monge Gómez, G. \& García Solano, J.A. (2006). La Cueva del Ángel (lucena, córdoba), a site of the middle and early Pleistocene in the south of the Iberian Peninsu1a. Trabajos de Prehistoria, 63 (2): 153-165. doi:10.3989/tp.2006.v63.i2.22

Dearing, J.A.; Dann, R.J.L.; Hay, K.; Lees, J.A.; Loveland, P.J.; Maher, B.A. \& O'Grady, K. (1996). Frequency- dependent susceptibility measurements of environmental materials. Geophysical Journal International, 124: 228-240. doi:10.1111/j.1365-246X.1996.tb06366.x

Dearing, J. (1999). Environmental Magnetic Susceptibility Using the Bartington MS2 System. British Library Cataloguing in Publication Data, 54 pp.

Deng, C.L.; Zhu, R.X.; Verosub, K.L.; Singer, M.J. \& Yuan, B.Y. (2000). Paleoclimatic significance of the temperature-dependent susceptibility of Holocene loess along a NW-SE transect in the Chinese loess plateau. Geophysical Research Letters, 27(22): 3715-3718. doi:10.1029/2000GL008462

Djerrab, A. \& Camps, P. (2011). Propriétés magnétiques du remplissage de la Caverna delle Manie (Finale Ligurie, Italie). ArcheoSciences, 34: 81-96.

Djerrab, A. \& Aïfa, T. (2010a). Stratigraphy and Palaeoenvironment of the Karaïn Cave Infill, Antalya, Turkey. Rock Magnetic Investigations. Studia Geophysica et Geodaetica, 54: 49-76. doi:10.1007/s11200-010-0003-0

Djerrab, A. \& Hedley, I. (2010b). Etude des minéraux magnétiques du site préhistorique de la Caverna delle Fate (Finale Ligurie, Savona, Italie). Quaternaire, 21 (2): 265-280.

Dunlop, D.J. \& Özdemir Ö. (1997). Rock Magnetism: Fundamentals and Frontiers. Cambridge University Press, New York, London and Cambridge, 573 pp. doi:10.1017/CBO9780511612794

Egli, R. (2003). Analysis of the field dependence of remanent magnetization curves. Journal of Geophysical Research, 108 (B2), 2081, doi:10.1029/2002JB002023

Evans, M.E. \& Heller, F. (2003). Environmental magnetism. Principles and applications of environmagnetics. Academic Press, 299 pp.

Eyre, J.K. (1997). Frequency-dependence of magnetic susceptibility for populations of single-domain grains. Geophysical Journal International, 129: 209-211. doi:10.1111/j.1365-246X.1997.tb00951.x

Geiss, C.E. \& Zanner C.W. (2006). Sediment magnetic signature of climate in modern loessic soils from the Great Plains. Quaternary International, doi:10.1016/j.quaint.10.035.

Guo, B.; Zhu, R.; Bai, L. \& Florindo, F. (2001). Rock magnetic properties of a loess-paleosol couple along an $\mathrm{N}-\mathrm{S}$ transect in the Chinese Loess Plateau. Science in China (Series D), 44 (12): 1099 - 1109. doi:10.1007/BF02906866

Hartstra, R.L. (1982). A comparative study of the ARM and Isr of some natural magnetites of MD and PSD grain size. Geophysical Journal of the Royal Astronomical Society, 71 (2): 497 - 518. doi:10.1111/j.1365246X.1982.tb05999.x

Heslop, D.; Dekkers, M.J.; Kruiver, P.P. \& Van Oorschot, I.H.M. (2002). Analysis of isothermal remanent magnetisation acquisition curves using the expectation-maximisation algorithm. Geophysical Journal International, 148: 58-64. doi:10.1046/j.0956-540x.2001.01558.x

Huet, A.M. (2003). Étude stratigraphique et sédimentologique de la Cueva del Ángel, province de Cordoue, Espagne. Mémoire de D.E.A. de Muséum National d'Histoire Naturelle, Paris, 27 pp. 
IGME, (1991). “Mapa Geológico de España”. Hoja 989, 17- 40. Madrid.

Kruiver, P.P.; Dekkers, M.J. \& Heslop, D. (2001). Quantification of magnetic coercivity components by the analysis of acquisition curves of isothermal remanent magnetisation. Earth and Planetary Science Letters, 189: 269-276. doi:10.1016/S0012-821X(01)00367-3

Kruiver, P.P.; Langereis, C.G.; Dekkers, M.J. \& Krijgsman, W. (2003). Rock-magnetic properties of multicomponent natural remanent magnetisation in alluvial red beds (NE Spain). Geophysical Journal International, 153: 317-332. doi:10.1046/j.1365-246X.2003.01880.x

Lanci, L.; Hirt, A.M.; Lowrie, W.; Lotter, A.F.; Lemcke, G. \& Sturm, M. (1999). Mineral-magnetic record of Late Quaternary climatic changes in a high Alpine lake. Earth and Planetary Science Letters, 170: 49-59. doi:10.1016/S0012-821X(99)00098-9

Le Borgne, E. (1960). Influence du feu sur les propriétés magnétiques du sol sur celles du schiste et du granite. Annales de Géophysique, 16: 159-196.

Maher, B.A. (1986). Characterisation of soils by mineral magnetic measurements. Physics of the Earth and Planetary Interiors, 42: 76-92. doi:10.1016/S00319201(86)80010-3

Maher, B.A. (1988). Magnetic properties of some synthetic sub-micron magnetites. Geophysical Journal of the Royal Astronomical Society, 94: 83-96. doi:10.1111/j.1365-246X.1988.tb03429.x

Marshall, A. (1998). Visualising burnt areas; patterns of magnetic susceptibility at Guiting Power 1 Round Barrow (Glos., UK). Archaeological Prospection, 5: 159- 177 . doi: $10.1002 /($ SICI) 1099 . 0763(199809)5:3<159::AID-ARP104>3.0.CO;2-D

Mullins, C.E. (1973). Magnetic viscosity, quadrature susceptibility, and frequency dependence of susceptibility in single-domain assemblies of magnetite and maghemite. Journal of Geophysical Research, 78 (5): 804-809. doi:10.1029/JB078i005p00804

Mullins, C.E. (1977). Magnetic susceptibility of the soil and its significance in soil science - a review. European Journal of Soil Science, 28: 223-246. doi:10.1111/j.1365-2389.1977.tb02232.x

Özdemir, Ö. \& Dunlop, D.J. (2000). Intermediate magnetite formation during dehydration of goethite. Earth and Planetary Science Letters, 177: 59-67. doi:10.1016/S0012-821X(00)00032-7

Özdemir, Ö. \& Dunlop, D.J. (2010). Hallmarks of maghemitization in low-temperature remanence cycling of partially oxidized magnetite nanoparticles. Journal of Geophysical Research, 115: B02101, 10 PP., doi: 10.1029/2009JB006756. doi:10.1029/2009JB006756
Peters, C., \& Thompson, R. (1998a). Supermagnetic enhancement, superparamagnetism and archaeological soils. Geoarchaeology, 13: 401- 413.

Peters, C. \& Thompson, R. (1998b). Magnetic identification of selected natural iron oxides and sulphides. Journal of Magnetism and Magnetic Materials, 183: 365374. doi:10.1016/S0304-8853(97)01097-4

Robertson, D.J. \& France, D.E. (1994). Discrimination of remanence-carrying minerals in mixtures, using isothermal remanent magnetization acquisition curves. Physics of the Earth and Planetary Interiors, 82 (22): 223-234. doi:10.1016/0031-9201(94)90074-4

Schmidbauer, E. \& Schembera, N. (1987). Magnetic hysteresis properties and anhysteretic remanent magnetization of spherical $\mathrm{Fe} 3 \mathrm{O} 4$ particles in the grain size range 60-160 nm. Physics of the Earth and Planetary Interiors, 46 (1-3): 77-83. doi:10.1016/00319201(87)90173-7

Tite, M. S., \& Mullins, C. E. (1971). Enhancement of the magnetic susceptibility of soils on archaeological sites. Archaeometry 13: 209-219. doi:10.1111/j.14754754.1971.tb00043.x

Verosub, K.L.; Fine, P.; Singer, M.J. \& Tenpas, J. (1993). Pedogenesis and paleoclimate: Interpretation of the magnetic susceptibility record of Chinese loess-paleosol sequences. Geology, 21:1011-1014. doi:10.1130/00917613(1993)021<1011:PAPIOT>2.3.CO;2

Verosub, K.L. \& Roberts, A.P. (1995). Environmental magnetism-past, present and future. Journal of Geophysical Research, 100 (B2), 2175-2192. doi:10.1029/94JB02713

Weston, D.G. (2004). The influence of waterlogging upon resultant susceptibilities: a series of laboratory reconstructions. Archaeological Prospection, 11: 107-120. doi:10.1002/arp.230

Worm, H.U. (1998). On the superparamagnetic-stable single domain transition for magnetite, and frequency dependency of susceptibility. Geophysical Journal International, 133: 201-206. doi:10.1046/j.1365246X.1998.1331468.x

Zouhair, S. (1996). Intérêt de la datation des planchers stalagmitiques par les méthodes fondées sur le déséquilibre dans les familles de l'uranium (230Th/234U). Application à quelques sites préhistoriques. Mémoire de D.E.A du Muséum National d’Histoire Naturelle. Paris.

Recibido el 7 de febrero de 2012 Aceptado el 6 de noviembre de 2012 Publicado online el 24 de abril de 2013 